\title{
PROCESOS SOCIALES Y DEGRADACIÓN AMBIENTAL: DEBATES RECIENTES EN EL ECO-SOCIALISMO
}

\author{
Social processes and environmental degradation: \\ recent discussions in eco-socialism
}

Sergio O. Sapkus*

\section{Resumen}

Surgida hace tres décadas, la corriente de pensamiento ambientalista conocida como ecosocialismo ha atravesado dos momentos. En el primero, sus impulsores intentaron injertar la teoría verde dentro del marxismo, o viceversa, aunque sin profundizar en el ajuste teórico que permitiera integrar estas dos vertientes teóricas. Como resultado, se elevaron críticas que señalaban el carácter $a d$ hoc de las fórmulas elaboradas para acortar las distancias entre ellas. Se fue abriendo entonces una segunda etapa, en la cual los analistas buscan entender de manera más exhaustiva el contexto ecológico del materialismo marxiano y, de esta manera, alcanzar una síntesis conceptual más profunda entre el ecologismo y el socialismo. El presente trabajo se ubica en esta perspectiva y comenta, con el fin de aportar al esclarecimiento teórico de las relaciones entre procesos sociales y deterioro ambiental, algunos debates recientes entablados entre autores eco-socialistas, poniendo especial énfasis en la noción de crisis en las condiciones ecológicas del desarrollo humano.

$<$ eco-socialismo $><$ degradación ambiental $><$ procesos sociales $><$ desarrollo humano $>$

\begin{abstract}
Having emerged three decades ago, the school of environmental thought named eco-socialism has gone through two moments. During the first of these, their proponents attempted to graft the green theory into Marxism, even though they did not delve into the theoretical framing that would allow the integration of these theoretical trends. As a consequence, critical voices appeared pointing out the ad-hoc nature of the formulae that had been devised in order to narrow the gap between them. A second phase was opened up, the scholars are now trying to grasp more comprehensively the ecological background of Marxian materialism, and to achieve a deep conceptual synthesis between ecologism and socialism. This paper has been drawn up with these considerations in mind, and it comments on some recent discussions between eco-socialists writers in order to improving the theoretical understanding of the relations between social processes and environmental degradation, whereas it puts a particular emphasis on the notion of crisis of the ecological conditions for human development.
\end{abstract}

$<$ eco-socialism $><$ environmental degradation $><$ social processes $><$ human development $>$

Recibido: 28/10/2015 // Aceptado: 04/12/2015

\footnotetext{
* Magister en Antropología Social, Profesor Titular, Facultad de Humanidades-Universidad Nacional de Formosa.sosapkus@yahoo.com.ar
} 


\section{Introducción}

Como es sabido, la década del sesenta marca el inicio de la preocupación pública generalizada acerca de la degradación ambiental en los países capitalistas centrales, aunque costó una década más de agitación tenaz sobre temas como las consecuencias dañinas del uso de pesticidas, los peligros inherentes e incontrolables de las plantas atómicas, los basureros de basura tóxica, $\mathrm{y}$, en términos más generales, los problemas derivados del desarrollo industrial/capitalista a gran escala, para que una "crisis ambiental" se reconozca como un tema de inquietud local, nacional e internacional. A partir de entonces se desarrollan dos procesos, conectados pero analíticamente distinguibles, a los que el sociólogo Frederick Buttel (1992) llama ecologización ("greening") y ambientalización ("environmentalization").

El primero es un fenómeno "ideológico o simbólico y una respuesta a la destrucción ambiental” (Ibíd: 2) que consiste en la divulgación de las preocupaciones ambientales y el aumento del ascendiente de los símbolos ambientales en el discurso social. El segundo está formado por "la expresión concreta de la amplia fuerza de la ecologización en las prácticas institucionales" (Ibíd.), esto es, su incorporación a ámbitos políticos y económicos de decisión, como así también en los círculos de la investigación científica. Estos procesos combinados de ecologización y ambientalización han puesto el tema verde en la agenda de los problemas políticos y sociales de finales del siglo XX y lo que va del presente.

En los inicios del proceso de ecologización, en los sesenta, se percibían los problemas ambientales esencialmente como una cuestión de una mala -o ausenteplanificación y de una injusta distribución de los “bienes" (por ej. adecuada infraestructura urbana) y "males" ambientales (por ej. contaminación). En tal sentido, el incipiente movimiento ecologista, que transitaba su "primera ola" (Eckersley, 1992), aparecía como un componente del movimiento más amplio que perseguía la justicia distributiva y una mayor participación en la toma de decisiones -en este caso en relación al uso de la tierra y los recursos naturales-; objetivos no alejados de los que ha sostenido históricamente la izquierda (en particular de la "nueva" izquierda que en ese momento ampliaba su abanico de intereses para incorporar temas hasta ese momento desestimados por la izquierda clásica, como el feminismo y la cuestión étnico/racial). A principios de los setenta, empero, la configuración de una "segunda ola" ecologista modificó este cuadro $\mathrm{y}$ dio origen a las divergencias entre el pensamiento verde-ecologista y el pensamiento rojo-izquierdista.

En efecto, por aquella década aparecen obras ecologistas que asumen abiertamente una postura neo-malthusiana, señalando que, debido a que existen "límites naturales" al crecimiento económico, se yergue una incompatibilidad insalvable entre el crecimiento demográfico y el aumento del bienestar humano. ${ }^{1}$ La problemática ambiental se entendía ahora, desde esta mirada, como "crisis de supervivencia" (Eckersley, op.

\footnotetext{
'El giro está marcado por dos trabajos publicados en 1968. Por un lado, el artículo de Garrett Hardin sobre la "tragedia de los comunes", aparecido en la revista Science (Hardin, 1968). Por el otro, el libro de Paul Ehrlich The population bomb (Ehrlich, 1968).
} 
cit.). Las implicaciones claramente conservadoras de estas posturas están reñidas con las intenciones de eliminar las desigualdades sociales, y particularmente, la pobreza, que anima a la izquierda. Así, el discurso verde adoptaba, desde la mirada izquierdista, una forma manifiestamente insensible frente a las aspiraciones legítimas de los pobres y los desposeídos de mejorar su estándar de vida, y por ende, no constituiría más que una nueva formulación de la ideología de las clases dominantes por perpetuar, enmascarándolos, sus privilegios. A su vez, la manera en que la izquierda tradicionalmente había perseguido la justicia social -mediante el crecimiento económico- aparecía ahora ante los verdes como claramente expoliadora ecológicamente. Desde esta perspectiva, a su vez, el discurso rojo no era más que una versión corrida a la izquierda de la ambición prometeica moderno-industrial de sojuzgar a la naturaleza e imponerle demandas excesivas. Se delinearon entonces los ejes básicos de la confrontación entre las corrientes verdes y las corrientes rojas referida a la relación entre la justicia social y el cuidado ecológico, oposición que, para muchos, perdura hasta el presente.

Pero si bien la respuesta hostil y defensiva primó entre la izquierda en los primeros momentos de los setenta, paulatinamente comenzó a perfilarse una tendencia que reconocía la legitimidad de los problemas que plantea el discurso verde. Comenzó a aceptarse la idea de que existen "límites materiales" a la producción capitalista, los cuales deben ser tomados seriamente en cuenta en el análisis social y la estrategia política de la izquierda. ${ }^{2}$ Esta idea, la necesidad de acercar y sintetizar los planteos verdes y los planteos marxistas, y más específicamente "leer a Marx como un crítico real o potencial de las consecuencias ambientales del capitalismo" (Castree, 2000: 18), configura la base a partir de la cual, ya en los ochenta, se configura el "eco-socialismo" o "eco-marxismo" como una corriente distinguible, en términos académicos y políticos, dentro del mundo verde-rojo en general.

Para acercar las posturas ecologistas y marxistas los autores eco-socialistas atacaron, desde entonces, cuatro áreas distintas de indagación. Por un lado, la problematización y desarrollo de las categorías heredadas del materialismo histórico en pos de ofrecer una exposición explicativa de la producción de la degradación ecológica. En segundo término, la redefinición del proyecto socialista para dar lugar a las dimensiones ecológicas. En tercer lugar, abordar los problemas de la filosofía ambientalista desde la perspectiva marxista; y en cuarto lugar, problematizar la cuestión de la agencia y la estrategia para una política emancipadora que incluya explícitamente la dimensión ambiental (Benton, 1996a).

Ahora bien, la manera en que han sido abordados estos asuntos y en que se ha concebido la confluencia entre los abordajes verdes y rojos no ha sido uniforme entre los eco-socialistas, por lo que se han ido perfilando diversas posiciones entre ellos. Como

\footnotetext{
2 El conocido analista marxista Raymond Williams encarna la actitud de esta corriente al plantear: "Esto es lo que cualquier socialista debe reconocer: existen límites materiales reales al modo de producción existente y a las condiciones sociales que forja" (1989: 221). Más adelante agrega en tal sentido que "el socialismo genuino puede conectarse con las apreciaciones racionales de la ecología. Tenemos que construir sobre la base del argumento socialista de que el crecimiento productivo, como tal, no acaba con la pobreza" (1989: 221).
} 
parte de una línea de investigación que venimos desarrollando desde hace un tiempo, en este trabajo quisiéramos indagar algunos de estos debates a fin de esclarecer conceptual y teóricamente abordajes de índole más empíricos sobre procesos socio-ambientales en el NEA, y específicamente en la provincia de Formosa. ${ }^{3}$ Particularmente quisiéramos comentar la importancia de la distinción entre una crisis ecológica entendida como perturbaciones en la dinámica económica del capital y una crisis ecológica entendía como trastornos multidimensionales de prácticas sociales y frustración de propósitos humanos, o, para utilizar la expresión de los autores que comentaremos, de degradación de las condiciones ecológicas del desarrollo humano. Para ello vamos a concentrarnos en la primera área de indagación mencionada más arriba, esto es, en la clarificación y desarrollo categorías de las categorías teóricas del materialismo histórico. De manera complementaria, también haré alguna mención a la cuarta área, la de la agencia del cambio social.

\section{Eco-socialismo: dos momentos}

Existen diversas maneras de distinguir posiciones dentro del eco-socialismo. Una de ellas traza la línea de separación siguiendo la cesura establecida entre las posturas "realistas" y las posturas "constructivistas" en el ámbito más amplio de las ciencias sociales que lidian con los temas ambientales. Desde este eje de delimitación se separa a los que sostienen que el ambiente natural es externo a la sociedad y constituye un dominio independiente de la realidad (realistas) de los que argumentan que tanto el "ambiente" y la "naturaleza" son construcciones de la sociedad, enfatizando el rol constitutivo que juega la cultura humana en la creación de la "verdad" o la "realidad" y ubicándose en tal sentido en una postura más bien agnóstica en relación a la realidad de los problemas ambientales (constructivistas). ${ }^{4}$ Entre los autores eco-socialistas son los geógrafos David Harvey y Neil Smith quienes han desarrollado una sofisticada aproximación marxista a los problemas ambientales desde una perspectiva globalmente "constructivista". 5

Dicho esto, nuestro interés en el presente ensayo es más acotado; nos interesa identificar y comentar dos momentos dentro de la vertiente genéricamente "realista". El criterio que tomamos para la discriminación de las dos hebras dentro de esta tendencia del pensamiento eco-socialista, siguiendo a Foster (2000) y Humphrey (2002), es el

\footnotetext{
Algunos resultados de esta indagación han aparecido en Sapkus 2007 y 2010.

4 Ver, por ej. Dunlap (2010) para una defensa reciente, desde la sociología, del realismo, y Hannigan (2007) y Demeritt (2002) para defensas, desde la sociología y la geografía respectivamente, del constructivismo.

5 Liodakis (2001) y Wallis (2008) ligan explícitamente a Smith (1984) y, en menor medida, a Harvey (1996) con el constructivismo. Liodakis incluso llega a acusarlos de seguir "un curso altamente idealista, con reminiscencias del abordaje neo-hegeliano que pierde la base ontológica materialista de un abordaje verdaderamente materialista" (Ibíd.: 115), con lo cual, agrega, se cae que en "un tipo paradójico de marxismo laissez faire" (Ibíd.). Una acusación similar esgrime Wallis, para quien el abordaje "posmoderno" de Smith lo ubica en una perspectiva que disculpa a la burguesía del daño ecológico. Una clasificación similar, aunque con una mirada más favorable al "constructivismo" de Smith/Harvey y que objeta a los "realistas" por su "positivismo" y "dualismo" se puede encontrar en FitzZimmons \& Goodman (1998).
} 
posicionamiento de los autores seleccionados frente a la herencia clásica del marxismo $\mathrm{y}$, de allí, las claves teóricas que desarrollan para afrontar el análisis de los temas ambientales del presente y su posible vía de solución. ${ }^{6}$

Así, se puede identificar una primera formulación eco-socialista caracterizada por la búsqueda de una reconstrucción verde del marxismo mediante la atenuación de algunos de sus elementos tradicionales y la introducción de elementos nuevos en su andamiaje teórico. Los autores más destacados de esta corriente son el filósofo austríaco/ francés André Gorz y el sociólogo/economista norteamericano James O'Connor. ${ }^{7}$ El segundo momento, a su vez, intenta recuperar a un Marx verde/ecológico de los escritos originales de Marx y Engels. Paul Burkett, uno de sus representantes más conocidos y prolíficos, sintetizaba a fines de la década del noventa, época en que comienza a ganar notoriedad esta vertiente, la tesis central de esta postura: "el abordaje que hace Marx de las condiciones naturales posee una lógica interna, una coherencia y un poder analítico que todavía no han sido reconocidos, inclusive en la literatura marxista ecológica (o "eco-marxista")" (1999: 1). Además de Burkett, otros autores destacados en esta corriente son John Bellamy Foster y Jonathan Hughes. ${ }^{8}$

\subsection{El primer momento del eco-socialismo: James O'Connor y la segunda contradicción}

\section{del capitalismo}

La obra descollante dentro del eco-socialismo es, sin duda, la de James O'Connor, quien a partir de la década de los ochenta comenzó a elaborar una sofisticada teoría que intenta ajustar el discurso verde con los principios del materialismo histórico. Para O’Connor: "Marx y Engels no dejaron más que un modesto legado de economía ecológica o ecología política" (2001: 157) y en su obra encontramos importantes "lagunas teóricas" para dar cuenta de los problemas ecológico/ambientales (2001: 180-1). Su propuesta apunta entonces a enmendar esta carencia de la obra marxiana, agregándole nuevos elementos. El núcleo de ellos estriba en ampliar el abordaje clásico de la contradicción central del capitalismo, a saber, entre fuerzas productivas y relaciones de producción, con el reconocimiento de una segunda contradicción entre las fuerzas (y relaciones) de producción y lo que va llamar las "condiciones de producción". Es con la elaboración y desarrollo de estas categorías que O’Connor intenta capturar conceptualmente las relaciones del capitalismo con la naturaleza "externa" y por ende, los problemas ecológicos.

El punto de partida de su argumentación es la proposición clásica marxista acerca de que el capitalismo es un sistema social afectado por crisis, esto es, un sistema que en su funcionamiento habitual genera barreras a su propio desarrollo futuro, que se manifiestan en forma de crisis económicas. Lo que subyace a estos desórdenes económicos es la dificultad de realizar la plusvalía generada por la explotación capitalista del trabajo. La

\footnotetext{
6 Otras maneras, además de las mencionadas, de clasificar corrientes eco-marxistas pueden encontrarse, entre otros, en Eckersley (1992), Benton (1996b) y Fine (2005).

7 Ver Gorz, 1980 y O’Connor, 2001.

8 Ver Burkett, 1999; Foster, 1999, 2004; y Hughes, 2000.
} 
competencia inter-capitalista, al obligar a los empresarios a elevar al máximo la tasa de explotación y mantener deprimidos los salarios, provoca el efecto inesperado de que los empresarios no puedan vender sus productos y obtener la ganancia contenida en ellas, desencadenándose periódicamente crisis de sobreproducción o de realización (exceso de producto en relación a la demanda existente). ${ }^{9}$ Desde un ángulo más teórico, esta tendencia a la sobreproducción, típica de la dinámica económica capitalista, no es otra cosa que el resultado de la contradicción central que anida en el sistema capitalista: el conflicto entre la producción socializada y la apropiación privada. O’Connor denomina "primera contradicción" a esta incompatibilidad. ${ }^{10}$

En el plano socio-político, la primera contradicción y su resultado concomitante, una explotación creciente de los trabajadores, estimula la lucha y la organización creciente de los obreros y la emergencia del movimiento obrero organizado. Dependiendo de la fortaleza y la dirección política de este movimiento, entre otros factores contingentes, las crisis recurrentes de la economía capitalista pueden dar lugar a su superación en un ordenamiento social superador o a la reestructuración de las relaciones capitalistas. Aunque el dominio del capital no sea desafiado exitosamente durante el tembladeral social y el aumento de la conflictividad social que acompañan las crisis, empero, los representantes del capital se ven obligados a tomar medidas para paliarla; medidas que implican una mayor socialización de la producción. ${ }^{11}$

La primera contradicción del capitalismo, entonces, erige barreras económicas y sociales que dificultan el proceso de acumulación del capital.

Ahora bien, aunque con una formulación de la dinámica de la crisis económica capitalista y el significado de la expresión marxiana de los "límites del capital" que puede ser discutida en los círculos marxistas, ${ }^{12}$ lo dicho hasta ahora no introduce ideas nuevas en el esquema conceptual marxiano. Donde O’Connor comienza a moverse por un andarivel más novedoso es cuando afronta el problema de la crisis ecológica. Para él, la crisis ecológica revela una época en el desarrollo capitalista signada por la aparición de nuevas barreras a la acumulación capitalista y por ende nuevas formas de crisis sistémicas. En otras palabras, se hace presente una nueva contradicción: la "segunda contradicción", cuya comprensión requiere el desarrollo de nuevas categorías de análisis, relativamente ausentes, o solamente implícitas en las obras originales de Marx. Entre ellas, como adelantamos, la central es la de condiciones de producción.

9 En palabras del propio O'Connor: "esto significa que la capacidad productiva tiene a aumentar más rápido que la demanda efectiva de mercancías (realización del capital), deteniendo la acumulación de capital o sumiendo a la economía en una recesión o depresión" (2001: 218)

${ }^{10}$ Manifestación específicamente capitalista, a su vez, del antagonismo entre las fuerzas productivas y las relaciones de producción que la teoría de la historia marxista clásica ha identificado como la fuerza motora del desarrollo histórico.

11 “....sabemos que el movimiento sindical 'empujó’ al capitalismo hacia formas más sociales de fuerzas y relaciones productivas, por ejemplo la negociación colectiva” (2001: 207).

${ }^{12}$ Nos referimos a que O' Connor defiende una interpretación "subconsumista" de la crisis económica, postura que si bien es compartida por buen parte de los economistas marxistas, está lejos de ser defendida unánimemente por los economistas de esa filiación. 
La noción de condiciones de producción alude a la capacidad que tienen las relaciones sociales capitalistas de perpetuarse, o, en otras palabras, pone el foco en aquello que asegura la existencia continua del capital como relación social. Los marxistas han estudiado tradicionalmente este problema mediante la noción de "reproducción social" del capital y, antes de la aparición del eco-socialismo, tomaban en consideración aspectos internos al mundo social. ${ }^{13}$ Lo que los eco-socialistas, con O'Connor a la cabeza, comienzan a teorizar son las formas de crisis social que derivan de las patrones típicos de interacción entre sociedades humanas dominadas por el capitalismo y sus condiciones externas (la "naturaleza").

O’Connor, recurriendo a las ideas del historiador económico húngaro no marxista Karl Polanyi, define a una condición de producción como "todo aquello que no se produce como una mercancía de acuerdo con la ley del valor o con las fuerzas del mercado, pero que el capital trata como si fuera una mercancía" (2001: 356-7). ${ }^{14}$ Según O'Connor, a Marx no se le había pasado por alto que la producción capitalista presuponía condiciones que no eran producidas como mercancías, y añade que, inclusive, "Marx definió tres clases de condiciones de producción. La primera son las 'condiciones físicas externas', o elementos naturales que intervienen en el capital constante y el variable. Segundo, la 'fuerza de trabajo' de los trabajadores se definió como las 'condiciones personales de producción'. Tercero, Marx se refirió a 'las condiciones comunales, generales, de la producción social', por ejemplo, los 'medios de comunicación' (2001 195). Pero, dicho esto, el pensador alemán nunca desarrolló todas las implicaciones analíticas de esta constatación. O'Connor "actualiza" y sistematiza estas intuiciones de Marx:

"En la actualidad las 'condiciones físicas externas' se analizan en términos de la viabilidad de los ecosistemas, los niveles adecuados de ozono atmosférico, la estabilidad de las líneas costeras y las cuencas, la calidad del suelo, el aire y el agua, y cosas por el estilo. La 'fuerza de trabajo' se discute en términos de bienestar físico y mental de los trabajadores, la clase y el grado de socialización de los mismos y los seres humanos, en general, como fuerzas productivas sociales y organismo biológicos. Las 'condiciones comunales' se analizan en términos del 'capital social', la 'infraestructura', y así sucesivamente (incluyendo, desde hace muy poco, el 'capital comunitario')" (2001)

\footnotetext{
${ }^{13}$ En los setentas y ochentas se incrementaron los estudios marxistas que abordaban el rol del Estado en la resolución (o profundización) de las crisis capitalistas. El propio O'Connor fue un activo participante de esta renovada área de indagación (ver O’Connor, 1981, 1984).

${ }^{14}$ Ver Polanyi, 1989.
} 
O'Connor argumenta que el despliegue de la economía capitalista tiende a degradar estas condiciones de producción que no son producidas como mercancías afectando así las posibilidades de reproducción social del sistema en su conjunto. Esto acontece porque los empresarios capitalistas individuales, al buscar mantener o restaurar su rentabilidad, se ven compelidos a rebajar los costos de sus empresas sin tener en cuenta el mantenimiento de las condiciones materiales y sociales de su propia producción. La lógica de la obtención de ganancias en el corto plazo, entonces, termina degradando, o por lo menos no conservando adecuadamente, esas condiciones. Esta dinámica conlleva el descuido de "[por ejemplo] las condiciones de trabajo (con lo cual se elevan los costos por salud), degradando los suelos (y reduciendo así la productividad de la tierra), o cerrando los ojos frente a la infraestructura urbana en deterioro (por lo cual se incrementa la congestión o los costos de vigilancia)" (2001: 286).

Todo esto exige la reparación de las condiciones de producción para que el proceso de acumulación del capital pueda perdurar. Dado que las condiciones de producción no son producidas como mercancías, esta tarea de reparación, como así también todo lo que tiene que ver con su suministro y regulación, es acometida por el Estado. Al final, esta intervención del Estado, vía aumento de la carga tributaria, incrementa los costos del conjunto del capital.

La elevación de los costos puede tener también otro origen más indirecto: la acción de los grupos sociales afectados en sus condiciones de vida y hábitat. Ciertamente, suele suceder que es la propia resistencia de las comunidades afectadas por episodios de contaminación o de otros efectos ambientales dañinos por el comportamiento de los empresarios individuales la que obliga al Estado a interceder para solucionar el problema. El resultado es el mismo que veníamos comentando: se incrementa el costo de las operaciones empresariales debido al maltrato, originado sistémicamente, de las condiciones de producción.

En suma, en su búsqueda de aumentar o restaurar sus ganancias los capitalistas individuales degradan las condiciones de producción, lo que termina aumentando los costos del capital en general. Se genera, entonces, una nueva contradicción. Una contradicción entre la acumulación de capital y las condiciones de producción. La primera contradicción, recordemos, era una crisis de sobre-producción. Ahora, en el caso de la segunda contradicción, se produce según O’Connor una crisis de subproducción. Esto significa, no tanto que se produzca una escasez material de materias primas, fuerza de trabajo o ambientes no dañados, sino, específicamente, que aumentan los costos de su adquisición, provocando, por el lado de los costos, un estrangulamiento en las ganancias.

No puede dejar de anotarse, como cuestión más general e ilustrativa de la novedad analítica que introduce el eco-socialismo en general, que O'Connor define a los problemas ecológicos de manera más amplia de lo que lo hacen a menudo las corrientes verdes. Al tomar en consideración a los sistemas naturales/ecológicos, el bienestar físico y mental humano, y la propia socialidad humana, se define al ambiente y los temas ambientales de manera que se incluyen los intereses de las mayorías. Quedan incorporados así asuntos tan variados que van desde la contaminación vehicular, 
pasando por seguridad en el trabajo hasta alcanzar, quizás de manera más importante aún, el desempleo y la pobreza. De este modo, los problemas básicos del socialismo son también problemas ambientales. Como señala el geógrafo David Pepper: "el ecosocialismo dice que tenemos que abordar la ecología desde la justicia social, y no al revés" (1993: 3).

Finalmente, O’Connor arriesga también una hipótesis atrevida concerniente a la agencia del cambio social progresivo. Siguiendo la analogía que establece entre la primera y la segunda contradicción dice que, así como aquélla engendraba un actor social, el movimiento obrero, que actuaba como freno social a la acumulación de capital $\mathrm{y}$, dadas ciertas circunstancias, podía reorganizar la sociedad sobre otras bases no capitalistas, así también la segunda contradicción engendra un tipo de actor social capaz de impulsar reordenamientos sociales progresivos y radicales. Aquí estamos hablando de los "nuevos movimientos sociales", los grupos humanos que, al ser atacados en sus condiciones de trabajo y de vida por la intrusión del capital, se organizan y ponen límites a tal entrometimiento:

...los nuevos movimientos sociales parecen tener un referente objetivo en las condiciones de producción: la ecología y el ambientalismo en las condiciones naturales; los movimientos urbanos del tipo que analizaron Manuel Castells y muchos otros en los setenta y principios de los ochenta en la infraestructura y el espacio urbanos, y movimientos tales como el feminismo, que se relaciona (entre otras cosas) con la definición de la fuerza de trabajo, la política del cuerpo, la distribución de la atención de los niños en el hogar, y cuestiones similares, en las "condiciones personales de producción” (2001: 358).

No sólo eso, O’Connor sugiere que esta disputa contenciosa por el respeto y la restauración de las condiciones de producción configura una vía diferente para llegar al socialismo. Así como existen según él, dos contradicciones, también existen dos caminos para llegar al socialismo: el del viejo movimiento obrero y el de los nuevos movimientos sociales ambientalistas.

Esta trinidad de conceptos: segunda contradicción, condiciones de producción y crisis de subproducción es, aun hoy, particularmente valiosa y fructífera para el análisis social de los temas ecológicos, a tal punto que el encuadre de O'Connor sigue siendo el más influyente entre los estudiosos marxistas o cercanos al marxismo (Castree, 2010: 1740). No obstante, si bien la propuesta de O'Connor ha constituye el punto de partida insoslayable de cualquier abordaje materialista/marxista del problema ambiental, se han detectado en ella algunas debilidades importantes. Pasaremos a tratar las ideas sustantivas de estas críticas. 
2.2. El segundo momento del eco-socialismo: las eco-credenciales de Marx y la crisis de las condiciones ecológicas del desarrollo humano

Las principales críticas dirigidas contra las tesis de O'Connor giran alrededor de dos cuestiones. Por un lado, se disputa la necesidad teórica de desarrollar una segunda contradicción y, de ese modo, el significado de la crisis del capitalismo y su lugar, allí, de la dimensión ecológica. Por otro lado, se discute el lugar de la clase social como agente de cambio social progresivo y su relación con los denominados "nuevos movimientos sociales", en particular el ecologista. Todo esto supone, también y por encima de todo, polemizar acerca del lugar que ocupa la obra de Marx y los clásicos marxistas en la elaboración de una propuesta eco-socialista coherente y penetrante.

En relación al primer punto, se objeta principalmente que se reduzca la crisis ecológica a una crisis de acumulación, esto es, a un problema de recesiones económicas y de descenso de ganancias e inversiones. Si bien uno de las contribuciones de O'Connor, que continúa el ángulo analítico de sus trabajos previos a su giro a la cuestión ecológica, reside en destacar la importancia de los factores no económicos en el examen del capitalismo, su propio análisis termina circunscribiendo el problema ecológico a una cuestión económico/productiva relativamente estrecha. Por otro lado, y aun desde el ángulo específico de la economía, su argumentación soslaya la posibilidad de que ciertas fracciones del capital puedan dedicarse a las actividades de mantenimiento ambiental y obtener una adecuada rentabilidad por ello, de modo tal que la restauración de las "condiciones de producción" contribuyan, más que socaven, la reproducción del capitalismo. Estas dos líneas de ataque a la obra eco-socialista de O'Connor revisten diversas formas, más o menos sofisticadas ${ }^{15}$, y aquí sólo nos detendremos en sus aspectos más relevantes.

Veamos. Según las ideas desarrolladas por O'Connor la degradación ecológica adquiere importancia en la medida en que afecta la rentabilidad del capital, sumándose, en este sentido, y por el lado de los "costos", a los mecanismos convencionales que causan trastornos e interrupciones en la reproducción del capital. Pero, afirma Michael Lebowitz en una crítica relativamente temprana a la idea de "la segunda contradicción":

\begin{abstract}
"más que las consecuencias no intencionadas de las acciones de los capitales individuales, en el núcleo de la 'segunda contradicción' encontramos que la esencia misma del capital consiste en determinar la naturaleza y el alcance de la producción sin tener en cuenta las necesidades humanas. Pero, esto es válido también para las condiciones subyacentes a la 'primera contradicción'. En un caso, existe una tendencia a producir sin considerar las condiciones naturales; en el otro, a producir sin considerar las condiciones sociales. Más que
\end{abstract}

\footnotetext{
${ }^{15}$ Este tipo de críticas lo desarrollan, entre otros, Benton (1996c), Lebowitz (1996), Burkett (1999), Spence (2000) y Strange (2000). Vlachou (2002) se destaca por ser la autora que más insiste en demostrar que la crisis ecológica representa una nueva fuente de rentabilidad del capital.
} 
dos contradicciones, existe sólo una: entre las necesidades del capital y las necesidades de los seres humanos" (1996: 228).

El puntapié inicial de Lebowitz, sugiriendo que la identificación de una "segunda contradicción" es innecesaria, va a ser continuado, profundizado y clarificado por otros autores y, a partir de allí, se modelarán las tesis centrales de la segunda ola eco-socialista.

El principal teórico de esta segunda ola es el economista norteamericano Paul Burkett. Burkett señala que la idea de la segunda contradicción se apoya en la presunción de que los gastos que el capital, a través del Estado, debe realizar para el mantenimiento y reparación de las condiciones naturales representan una canalización improductiva del plusvalor y por ende, a través una reducción de la demanda agregada, una caída en la rentabilidad empresarial. Pero, continúa Burkett, este postulado presenta varios problemas. Por un lado, reduce la cuestión ecológica al problema de la crisis económica, como así también pasa por alto la posibilidad de que el capitalismo pueda reproducirse a sí mismo apoyándose en dichas actividades de mantenimiento y reparación que son rentables. Así, Burkett destaca que "el control de la contaminación y el tratamiento del agua, las prisiones y los servicios de seguridad, todos ellos constituyen sectores muy rentables, aunque para muchos capitalistas individuales puedan representar costos privados y mayores impuestos" (1999: 195). De modo tal que, en suma, "La cuestión es que los 'costos externos' de la acumulación de capital crea oportunidades de ganancias para la producción y realización de plusvalía, no sólo para capitales individuales sino para el capital en su conjunto" (1999: 195).

Por el otro, y este es el punto que consideramos más relevante de las observaciones de Burkett, que esta reproducción del capitalismo puede llevarse a cabo "sin revertir las condiciones de degradación de la naturaleza desde la perspectiva de un desarrollo humano coevolucionario" (Burkett, 2007: 24). Aquí, se reprocha a O'Connor su subestimación de la capacidad del capitalismo de acumular un en ambientes degradados. Burkett abre, en tal sentido, una ventana que permite pensar al problema ambiental de un modo distinto. El problema ambiental no consistiría tanto, ni siquiera principalmente, en el debilitamiento del lucro capitalista que deriva del alza de los costos provocados por la reparación de los daños producidos por la producción pretérita. El problema en que el capitalismo perdure, en tanto y en cuanto la posibilidad de la rentabilidad se mantenga dentro de parámetros relativamente aceptables para los empresarios, pero que, en simultáneo, degradando constantemente las condiciones de vida de las personas comunes. La reflexión que cierra esta argumentación de Burkett es que "el capitalismo es capaz de funcionar sin cuidados de salud universales, asequibles y de calidad, y sin una fuerza de trabajo que está en buen estado de salud... todo lo que necesita es un suministro suficiente de fuerza de trabajo explotable" (2007: 36).

En este punto acierta el sociólogo Ted Benton (1996b) cuando señala que lo que teoriza O'Connor no es tanto la degradación ecológica en sí misma, sino las crisis ecológicas, entendiendo crisis en su sentido económico convencional. Así las cosas, para 
obtener explicaciones que aclaren los procesos más generales y abarcativos de deterioro ambiental, la lente de la segunda ola eco-socialista aporta categorías y observaciones penetrantes más útiles.

Burkett clarifica su propuesta delimitando dos maneras diferentes de entender la crisis ambiental: "es esencial distinguir la crisis ambiental de la acumulación de capital de la crisis ambiental en el sentido de deterioro general de las condiciones de desarrollo de los seres humanos como especie social y natural" (1999: 196, cursivas de Burkett). El autor insiste que este segundo tipo de crisis no implica automáticamente el primero. Por otra parte apunta que ambas crisis son consecuencia del capitalismo, son "síntomas" distintos de la crisis capitalista que actualiza la contradicción central en el capitalismo entre la riqueza entendida como valor y la riqueza entendida como valores de uso que permitan un desarrollo humano menos restringido y más sustentable -esto es, que permitan la satisfacción de las "necesidades del individuo social" (como opuesto al "mero trabajador") que desde las propias palabras de Marx, constituían el objetivo de una sociedad socialista (Marx, 1997, II: 232).

En un plano más abstracto, Burkett fundamenta esta distinción, a nuestro criterio fundamental, apelando a la teoría del valor de Marx, profundizando así la cesura entre las dos perspectivas eco-socialistas. El punto teórico central es que el avance capitalista no debe verse únicamente como una relación social, sino también como una relación material (seres humanos-naturaleza), e inclusive, como una "relación ecológica" (2007: 39-40).

En la medida que el capitalismo, despojando a los productores directos de sus condiciones de vida, subordina crecientemente la vida social a su lógica, la producción es gobernada cada vez más por la búsqueda de la ganancia monetaria y la evaluación monetaria de las cosas. Es la maximización del valor abstracto y no la producción de valores de uso cualitativamente diferentes como objetivo determinante del entrelazamiento de los seres humanos con la naturaleza lo que lleva a degradación del rol esencial de las condiciones naturales en la producción de la riqueza en general y del trabajador. En la base de ello está una contradicción creciente, potenciada por el proceso de mercantilización, entre las características de la moneda, esto es, su homogeneidad, su divisibilidad, su movilidad y su cantidad ilimitada, y las características de las condiciones naturales y los ecosistemas, que se caracterizan por su variabilidad cualitativa, por su interconexión (es decir, su divisibilidad limitada), su ubicación específica y su limitación cualitativa. De este modo el capitalismo, con su división social clasista del trabajo que aliena a los trabajadores de sus condiciones naturales de producción y mercantiliza la vida social y material, crea y profundiza con el tiempo una grieta "irreparable" en el metabolismo entre los seres humanos y la naturaleza; lo que ha sido llamado la "grieta metabólica". ${ }^{16}$

\footnotetext{
${ }^{16}$ La noción de grieta metabólica ha sido elaborada recientemente por John Bellamy Foster (1999, 2004) a partir de las sugerencias que brinda Marx (1991). Con esta noción se busca explicar los efectos negativos del desarrollo capitalista en el ambiente como resultado de la separación de la producción de su base biológica. La producción capitalista, se argumenta, fractura el balance de intercambios energéticos entre la naturaleza y la sociedad, produciéndose un "desgarramiento insanable en la continuidad del metabolismo
} 
En definitiva, para Burkett es innecesario y hasta engañoso sugerir que se necesita acoplar una "segunda crisis" a la primera contradicción que se expresa en las crisis de sobreproducción. Más bien, las dos contradicciones están unidas indisolublemente en la medida en que son manifestaciones de las tensiones básicas subyacentes en el funcionamiento del capitalismo en torno a la formación del valor. La primera contradicción hunde sus raíces en el poder social y político del capital sobre el trabajo. Y este poder, en sí mismo, está enraizado en la apropiación de las condiciones naturales y sociales.

Burkett y los autores adheridos a la segunda ola del eco-socialismo insisten en que la explicación teórica de la tendencia del capitalismo a provocar daño ecológico está contenida en las categorías que analizan las propias contradicciones del capital, sin necesidad de un aditamento verde. Estas categorías, por lo demás, están expuestas en los clásicos marxistas, particularmente en El Capital. Se desprenden de todo ello dos conclusiones. Por un lado, la refutación de los argumentos verdes acerca de la existencia de un Marx anti-ecológico, argumento aceptado en parte por la primera ola eco-socialista. Por otra parte, la concepción del proyecto intelectual eco-socialista como un desarrollo de esas categorías clásicas.

Antes de pasar al cierre, quisiéramos comentar rápidamente un segundo aspecto de las críticas a O'Connor que se ubican en la cuarta temática abordada por los eco-socialistas que había señalado al inicio de esta monografía (2001: 3). Así como se problematiza, e incluso se rechaza, la idea de una segunda contradicción, también se disputa la idea de una segunda vía al socialismo y, particularmente, la naturaleza política del movimiento ecologista. Aquí se pueden diferenciar dos tipos de comentarios. Por un lado, los que, como Burkett y Spence (op. cit.), defienden la idea de que la luchas ambientales puede ser entendida desde una perspectiva que conceptualice de manera más amplia la categoría "clase obrera" y sus intereses. Lo que estos autores intentan destacar es que, en concordancia con su refutación de la noción de segunda contradicción, no existe razón para conceptualizar a las protestas que emergen con la problemática ambiental como algo distinto a la lucha de clases y, particularmente, rechazan la sugerencia que O'Connor deja traslucir en varias oportunidades acerca de que los movimientos ecologistas serían "no de clase" (por ej. O'Connor, op. cit., 32).

\footnotetext{
social, prescrito por las leyes naturales de la vida, como consecuencia de lo cual se dilapida la fuerza del suelo, dilapidación ésta que, en virtud de comercio, se lleva mucho más allá de las fronteras del propio país" (Marx, 1991: 1034). La idea original de Marx plantea que la grieta metabólica es la consecuencia de una doble separación: de los productores directos y los medios de producción, por un lado; de la ciudad y el campo, por otro. A medida que el capitalismo se desarrolla en las ciudades, aumenta el flujo de personas que, desde el campo, se desplaza hacia ellas para emplearse como asalariados urbanos. Los productos agrarios también siguen este desplazamiento demográfico, ampliándose la distancia social y espacial entre la producción y el consumo. Como resultado, los nutrientes del suelo que eran reciclados en el sitio de producción son embarcados a las ciudades para ser consumidos por las nuevas clases urbanas. Los nutrientes que no son absorbidos por los cuerpos humanos son eliminados como desechos. En definitiva, estos nutrientes -al ser eliminados como residuos en los ámbitos urbanos- no vuelven a los lugares de producción agrícola, desgarrándose el ciclo de nutrientes del suelo y las relaciones humanosnaturaleza extra humana.
} 
Por otro lado, están los que, como Benton (1996c), se muestran recelosos ante la idea, que para algunos incluso llega a constituir la "sustancia real" del modelo de O’Connor (Henderson, 2009: 276), de que los movimientos ambientalistas son, objetivamente, anti-capitalistas. En este sentido, lo que recuerda Benton es que gran parte del movimiento ambientalista, al presionar a los capitales individuales para que adopten medidas "ambientalmente amigables" terminan promoviendo los intereses a largo plazo del capital en general al legitimar formas de suministro de "condiciones de producción" reparadas que renuevan y oxigenan al proceso de acumulación. En este sentido, la sola lucha contra los capitales individuales, sin un cuestionamiento sistémico al capitalismo, concluye Benton, contribuye más a la reproducción del capitalismo más que a su derrocamiento.

\section{Reflexiones finales}

La aparición del ecomarxismo o ecosocialismo abre una perspectiva teórica valiosa para abordar el problema de la relación entre los procesos sociales y la degradación ambiental, superadora de la "mistificación naturalista" (Gimenez, 2002) del discurso verde no marxista, encerrada en categorías como industrialismo y modernidad, que erróneamente hace aparecer las fuerza sociales como límites o causas naturales del daño ambiental. Al mismo tiempo, también permite resolver algunos problemas del enfoque tradicional de la izquierda que había desestimado estos problemas e interpretaba a Marx desde una perspectiva productivista/industrialista. Ahora bien, si bien el ecosocialismo consiste en leer a Marx como un crítico, potencial o real, de las consecuencias ambientales del capitalismo, no existe acuerdo entre sus representantes acerca del significado y la importancia ecológica de las categorías marxianas.

En tal sentido, se pueden distinguir dos grandes posturas, a las que he agrupado bajo los rótulos cronológicos de primer momento y segundo momento. La postura paradigmática, y aun la más influyente del ecosocialismo es la de James O'Connor que reconstruyó el aparato conceptual de la economía política marxiana para sensibilizarlo ecológicamente. La principal operación de O’Connor en tal sentido es la elaboración, cuyo núcleo pertenece más bien a Karl Polanyi, de la categoría de condiciones de producción, que hace referencia a todos aquellos procesos y entidades que el capital toma como mercancías aunque no son producida como tales. Allí se encuentra la naturaleza "externa". La lógica capitalista, dice O'Connor, lleva a destruir estas condiciones y, la propia necesidad de conservar estas condiciones conduce, a su vez al aumento de los costos de las materias primas y otros insumos usados por las empresas. Esto genera una crisis, a la que O'Connor llama segunda contradicción. A este análisis que busca cubrir huecos del arsenal teórico marxista, O’Connor añade que esta contradicción genera también un actor social que desafía al capitalismo y son los movimientos sociales afectados por la depredación de sus condiciones de vida (que forman parte de las condiciones de producción del capital).

La postura de los autores enrolados en el segundo momento cuestiona la idea de la segunda contradicción. Aquí podemos distinguir dos dimensiones de la crítica. Por un 
lado no se considera necesario recurrir a ideas ajenas de la obra marxiana y se considera que, si se mira más de cerca, se pueden descubrir en la obra de Marx las categorías teóricas para lidiar con las consecuencias ambientales negativas del capitalismo. En términos más amplios, y teniendo en cuenta la disputas entre verdes y rojos, la defensa y documentación de un Marx ecológicamente sensible es sumamente importante para el proyecto eco-socialista en general y se destaca, como ya hemos adelantado, como uno de los rasgos decisivos del "segundo momento". A partir de allí también se objeta la idea de dividir en dos a las contradicciones capitalistas ya que, se afirma, ambas constituyen un todo, de modo tal que la única contradicción se establece entre la producción para obtener ganancias monetarias y la producción para la satisfacción de las necesidades. El punto central es analizar la degradación ambiental poniendo como eje el problema de la satisfacción de las necesidades multidimensionales de los seres humanos, entendidos como "individuos sociales".

Si bien los contrastes pueden resultar exagerados y no deja de ser lícito ensayar algún tipo de reconciliación entre ambas posiciones (como intenta, por ejemplo, Perelman, 1999), consideramos que la riqueza analítica que abre la idea de condiciones ecológicas del desarrollo humano es sumamente esclarecedora y muestra fructíferas sendas de investigación de procesos concretos de despliegue de procesos sociales con implicaciones medioambientales negativas, estimadas desde el punto de vista de la satisfacción no restringida de necesidades humanas, que no están contenidas en la idea de la segunda contradicción. En tal sentido, abre vías de análisis y explicación de los trastornos socio-ambientales que han afrontado, y, gracias a las acciones de las elites económicas y estatales, siguen sufriendo de manera renovada, las poblaciones subalternas de una "periferia extrema" como la provincia de Formosa.

\section{Referencias bibliográficas}

Benton, T. 1996a. "Introduction to Part I". En: Ted Benton (ed.) The greening of Marxism. New York, Guilford, pp. 7-15.

Benton, T. 1996b. "Introduction to part II". En: Ted Benton (ed.) The greening of Marxism (cit.), pp. 103-04.

Benton, T. 1996c. "Introduction to part III". En: Ted Benton (ed.) The greening of Marxism (cit.), pp. 187-95.

Burkett, P. 1999. Marx and nature. A red and green perspective. New York, St Martin's Press.

Burkett, P. 2007. "Two stages of ecosocialism? Implications of some neglected analyses of ecological conflict and crisis". En: International Journal of Political Economy, Vol 35, No 3, pp 23-45.

Buttel, F. 1992. "Environmentalization: origins, processes, and implications for rural social change". En: Rural Sociology, Vol 57, No 1, pp 1-27.

Castree, N. 2000. "Marxism and the production of nature". En: Capital and Class, Vol 24, $\mathrm{N}^{\mathrm{o}} 3$, pp 5-36. 
Castree, N. 2010. "Neoliberalism and the biophysical environment 2". En: Geography Compass, Vol 4, № 12, pp. 1734-1746.

Demeritt, D. 2002. "What is the 'social construction of nature'? A tipology and sympathetic critique". En: Progress in Human Geography, Vol 26, No 6, pp. 76790.

Dunlap, R. 2010. "The maturation and diversification of environmental sociology: from constructivism and realism to agnosticism and pragmatism". En: Redclift, M. \& Woodgate, G. (eds.) The International Handbook of environmental sociology. 2 ed. Northampton, Edwar Elgar, pp. 15-32.

Eckersley, R. 1992. Environmentalism and political theory. London, UCL Press.

Ehrlich, P. 1968. The population bomb. New York, Buccaneer.

Fine, B. 2005. "From actor-network theory to political economy". En: Capitalism, Nature, Socialism, Vol. 16, No 4, pp. 91-108.

FitzZimmons, M. \& Goodman, D. 1998. "Incorporating nature”. En: Bruce Braun \& Noel

Castree (eds.) Remaking reality. London, Routledge, pp. 193-219.

Foster, J. B. 1999. "Marx's theory of metabolic rift: classical foundations for environmental sociology". En: The American Journal of Sociology, Vol. 105, № 2, pp.366-405.

Foster, J. B. 2000. "Environment Politics: Analyses and alternatives". En: Historical Materialism, Vol. 8, $\mathrm{N}^{\mathrm{o}}$ 1, pp. 461-7.

Foster, J. B. 2004. La ecología de Marx. Madrid, El Viejo Topo.

Gimenez, M. 2000. "Does ecology need Marx?" En: Organization and Environment, Vol. 13, N 3, pp. 292-304.

Gorz, A. 1980. Ecology and politics. London, Pluto Press.

Hannigan, J. 2006. Environmental sociology. 2 ed. New York, Routledge.

Hardin, G. 1968. "The tragedy of the commons", Science, Vol 12, No 3.859, pp. 1.243-48.

Harvey, D. 1996. Justice, nature and the geography of difference. Cambridge, Ma, Blackwell.

Henderson, G. 2009. "Marxist political economy and the environment". En: Noel Castree et al (eds.) A companion to environmental geography. Malden, WileyBlackwell, pp. 266-93.

Hughes, J. 2000. Ecology and historical materialism. Cambridge, Cambridge University Press.

Humphrey, M. 2002. Preservation versus the people? Oxford, Oxford University Press.

Lebowitz, M. 1996. “Capitalism: how many contradictions?” En: Ted Benton (ed.) The greening of Marxism (cit.), pp 226-28

Liodakis, G. 2001. "The people-nature relation and the historical significance of the Labour theory of value", Capital and Class, Vol 25, No 1, pp. 113-40.

Marx, K. 1991. El Capital. TI. México, Siglo XXI.

Marx, K. 1997. Elementos fundamentales para la crítica de la economía política (Grundrisse), 1857-1858. México, Siglo XXI. 3 T.

O’Connor, J. 1981. La crisis fiscal del Estado. Barcelona, Edición 62. 
O’Connor, J. 1984. Accumulation crisis. New York, Basil Blackwell.

O’Connor, J. 2001. Causas naturales. Ensayos de marxismo ecológico. México, Siglo XXI.

Pepper, D. 1993. Eco-socialism. From deep ecology to socialjustice. London, Routledge.

Perelman, M. 1999. "Mr. Natural”, Capitalism, Nature, Socialism, 10(4): 147-52.

Polanyi, K. 1989. La gran transformación. Madrid, Ediciones de la Piqueta.

Sapkus, S. 2007. "Capital, campesinos y medioambiente en Formosa. La resistencia campesina a las firmas productoras de soja transgénica", en Hocsman, L. D. (comp.) Transformaciones productivas e impactos sociales agrarios en años de neoliberalismo. Córdoba, Ferreira Editor-UNVM, pp. 99-120.

Sapkus, S. 2010. "La problemática ambiental. Algunos perspectivas desde la antropología", en Investigaciones y Ensayos Geográficos. Revista de Geografía, Vol VII, No 8 , pp. 25-32.

Smith, N. 1984. Uneven development. Nature, capital and the production of space. Oxford, Basil Blackwell.

Spence, M. 2000. "Capital against nature. James O'Connor's theory of the second contradiction of capitalism". En: Capital and Class, Vol 24, No 3, pp. 81-109.

Strange, G. 2000. "Capitalism, valorization and political economy of ecological crisis", Capital and Class, Vol 24, No 3, pp 55-80.

Vlachou, A. 2002. "Nature and value theory". En: Science and Society, Vol 66, No 2, pp $169-201$

Wallis, V. 2008. "Capitalist and socialist responses to the ecological crisis". En: Monthly Review, Vol. 60, N 6, pp. 25-40.

Williams, R. 1989. "Socialism and ecology”. En: Resources of hope. Culture, democracy, socialism. London, Verso, pp. 210-26. 
